\title{
Facile Formation of Unexpected [m,6,n]-tricyclic Spiranes via Intramolecular $[3+2]$ Cyclization of Platinum-bound Pyrylium with Alkenes ${ }^{\dagger}$
}

\author{
Chang Ho Oh, Sang Yong Tak, Ji Ho Lee, and Lanhua Piao \\ Department of Chemistry, Institute of Natural Science, Hanyang University, Seoul 133-791, Korea \\ *E-mail: changho@hanyang.ac.kr \\ Received March 13, 2011, Accepted April 1, 2011
}

\begin{abstract}
Enynals bearing an olefinic pendant were successfully cyclized via Huisgen-type [3+2] cycloaddition to the tetracyclic Pt-carbene complexes which would undergo insertion into a $\mathrm{C}$-H bond of the $\beta$-position to afford the fused cyclopropane intermediates. Their tandem rearrangement afforded diverse types of spiranes depending on the tethered alkenes of the enynals.
\end{abstract}

Key Words : Cyclization, Platinum, Pyrylium ions, Carbene, Spirane

\section{Introduction}

The cycloaddition reaction is one of the most powerful synthetic tools for the construction of various cyclic compounds. In particular, the intramolecular metal-catalyzed cycloaddition is very attractive because it gives multicyclic compounds from acyclic substrates in one pot. Among the various strategies for diverse polycyclic skeletons, intramolecular metal-catalyzed $[4+2$ or $3+2]$ cycloadditions are well established strategy for the synthesis of carbo- and heterocyclic structures, which have been reported. ${ }^{1}$ During the course of our scientific endeavors leading to a general and modular entry to polycyles, we reported a highly unique behavior of Pt-carbene complexes $\boldsymbol{A}$, formed via a Huisgen-type [3+2] cycloaddition between metal-bound pyrylium and a pendant alkene. ${ }^{2}$ In the cases involving a five-membered ring formation in the $3^{\text {rd }}$ cycle, we have reported Au-catalyzed cyclization of enynals (like 1) to yield 2,3,10,10a-tetrahydrobenzo[f]azulen-9(1H)-ones (like 2) via $[3+2]$ cycloaddition followed by elimination of $\mathrm{H}^{1}$

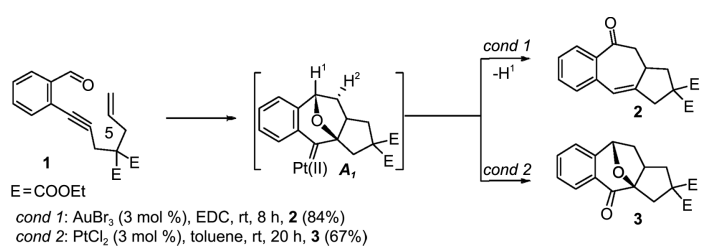

cond 1: $\mathrm{AuBr}_{3}(3 \mathrm{~mol} \%), \mathrm{EDC}, \mathrm{rt}, 8 \mathrm{~h}, \mathbf{2}(84 \%)$

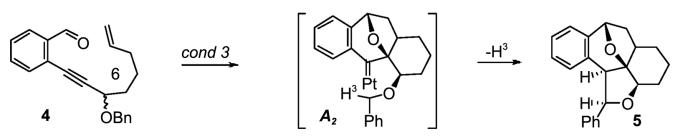

cond 3: $\mathrm{PtCl}_{2}\left(\mathrm{PPh}_{3}\right)_{2}(5 \mathrm{~mol} \%)$, toluene, $120^{\circ} \mathrm{C}, 4 \mathrm{~h}, 5(77 \%)$

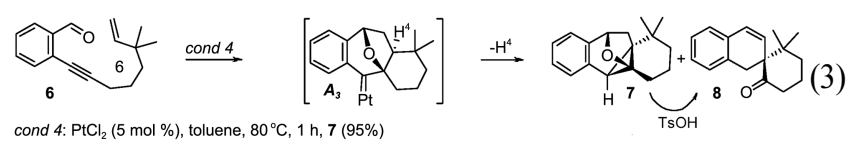

This paper is dedicated to Professor Eun Lee on the occasion of his honourable retirement.
[Eq. (1)]. ${ }^{3}$ It is noted that elimination of $\mathrm{H}^{2}$ from $\boldsymbol{A}_{\boldsymbol{I}}$ would result in sequential migration eventually to form the naphthalene derivative. ${ }^{4}$ Surprisingly, Pt-catalyzed cyclization of 1 would also form $\boldsymbol{A}_{\boldsymbol{I}}$ via [3+2] cycloaddition with an alkene and eventually resulted in $\mathbf{3}$ as a major product, presumably via oxygenation of $\boldsymbol{A}_{\boldsymbol{l}}{ }^{5}$ During our synthesis of tricycles containing a six-membered ring in the $3^{\text {rd }}$ cycle, we observed an unique insertion of the Pt-carbene intermediate $\boldsymbol{A}_{2}$, derived from 4, into the benzylic C-H of the $\delta$-position to afford 5 [Eq. (2)]. ${ }^{6}$ The Pt-carbene $\boldsymbol{A}_{3}$, without possessing such $\mathrm{CH}$ bond, resulted in insertion into the tertiary $\mathrm{CH}$ bond to form the cyclopropane ring like $7 .^{7}$ Furthermore, heating the reaction solution of $\mathbf{6}$ or $\mathrm{TsOH}$-catalyzed isomerization of the isolated 7 furnished 8 in high yield [Eq. (3)].8 It was worthwhile to note that the substrates like $\mathbf{6}$ with an aromatic anchor were successful in the present cyclopropanation of the Pt-carbene complex $\boldsymbol{A}_{\mathbf{3}}$.

We have examined cycloalkene-based substrates 9 in order to extend the scope of the Pt-catalyzed cyclizations. Thus, we have found that $\mathrm{H}^{5}$ in $\boldsymbol{A}_{\mathbf{4}}$ intermediate was involved in this sequence to furnish the eliminated products $\mathbf{1 0}$ (Scheme 1). ${ }^{9}$ In Pt-catalyzed cyclization of 2alkynylcycloalkenecarboxaldehydes 9a-g with a remote olefinic tether ( $\mathrm{n}=2$ or larger), we found a catalytic method forming the corresponding $[\mathrm{m}, 6, \mathrm{n}]$-tricyclic spiranes $\mathbf{1 1}$ in good to excellent yields.

\section{Result and Discussion}

We examined the reaction of substrate $9 \mathbf{a}$ in the presence of Pt catalysts under a variety of conditions (Table 1). First of all, the reaction of $9 \mathbf{a}$ with $\mathrm{PtCl}_{2}\left(\mathrm{PPh}_{3}\right)_{2}$ as the catalyst proceeded well under reflux in toluene to furnish products 10a and 11a in $80 \%$ and $10 \%$ yields, respectively (entry 7 ). When 9a was treated with $\mathrm{PtCl}_{2}\left(\mathrm{PPh}_{3}\right)_{2}$ at $80{ }^{\circ} \mathrm{C}$ in 1,2dichloroethane (EDC) for $12 \mathrm{~h}, \mathbf{1 0 a}$ was formed as a major product (entry 8). Several conditions were tested to optimize the reaction efficacy. This reaction was also successful in other solvents, such as $p$-dioxane (entry 9), and other 


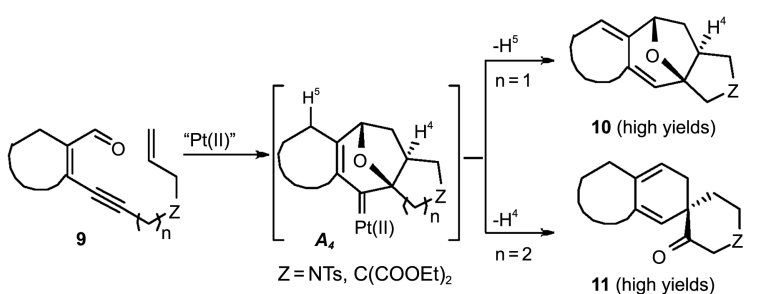

Scheme 1. Pt-catalyzed reaction of non aromatic substrate 9.

Table 1. Platinium-Catalyzed Reaction of Enynal 9a

\begin{tabular}{|c|c|c|c|c|}
\hline & $9 a$ & \multicolumn{2}{|c|}{ 10a } & 11a \\
\hline Entry & $\begin{array}{l}\text { Catalysts } \\
(5 \mathrm{~mol} \%)\end{array}$ & Solvent & $\begin{array}{c}\text { Temp }\left({ }^{\circ} \mathrm{C}\right) \\
\text { time }(\mathrm{h})\end{array}$ & $\begin{array}{l}\text { Products }^{b} \\
(\% \text { yield })^{\mathrm{c}}\end{array}$ \\
\hline 1 & $\mathrm{PtCl}_{2}$ & toluene & 120,1 & $11 \mathbf{a}(72)$ \\
\hline 2 & $\mathrm{PtCl}_{2}$ & toluene & 80,1 & 11a (91) \\
\hline 3 & $\mathrm{PtCl}_{2}$ & EDC & 60,5 & $11 \mathbf{a}(25)$ \\
\hline 4 & $\mathrm{PtCl}_{2}$ & $\mathrm{CH}_{3} \mathrm{CN}$ & 80,12 & $11 \mathbf{a}(20)$ \\
\hline 5 & $\mathrm{PtCI}_{2}$ & $p$-dioxane & 80,12 & 11a (55) \\
\hline 6 & $\mathrm{PtCI}_{2}\left(\mathrm{PPh}^{3}\right)^{2}$ & toluene & 100,24 & 10a $(70), 11 \mathbf{a}(15)$ \\
\hline 7 & $\mathrm{PtCl}_{2}\left(\mathrm{PPh}^{3}\right)^{2}$ & toluene & 120,12 & $10 \mathbf{a}(80), 11 \mathbf{a}(10)$ \\
\hline 8 & $\mathrm{PtCl}_{2}\left(\mathrm{PPh}^{3}\right)^{2}$ & EDC & 80,12 & $\mathbf{1 0 a}(43)$ \\
\hline 9 & $\mathrm{PtCI}_{2}\left(\mathrm{PPh}^{3}\right)^{2}$ & $p$-dioxane & 120,18 & 10a $(63), 11 \mathbf{a}(15)$ \\
\hline
\end{tabular}

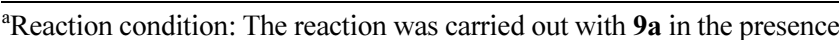
of $10 \mathrm{~mol} \%$ of the Pt catalyst under the given conditions. ${ }^{b} \mathrm{E}=$ COOEt. ${ }^{c} \mathrm{EDC}=1,2$-dichloroethane. ${ }^{\mathrm{d}}$ Isolated yields.

conditions, such as $100^{\circ} \mathrm{C}, 24 \mathrm{~h}$ (entry 6). Surprisingly, when 9a was treated with $\mathrm{PtCl}_{2}$ at $120{ }^{\circ} \mathrm{C}$ in toluene for $1 \mathrm{~h}$, an unexpected product 11a was isolated in $72 \%$ yield without forming product 10a (entry 1). The $\mathrm{PtCl}_{2}$ catalyst showed selective reactivity of Pt-carbene complex $\boldsymbol{B}_{4}$ which would undergo insertion into a $\mathrm{C}-\mathrm{H}^{5}$ bond of the $\beta$-position to afford 11a in high yield. This reaction was also working in other solvents such as 1,2-dichloroethane (EDC), $\mathrm{CH}_{3} \mathrm{CN}$, and $p$-dioxane (entries 3-5). Finally, we could optimize this reaction by decreasing temperature, where 11a was isolated in $91 \%$ yield (entry 2 ).

Various substrates were subjected to optimized reactions for the formation of 11a to explore the scope and limitation of this process (Figure 1).
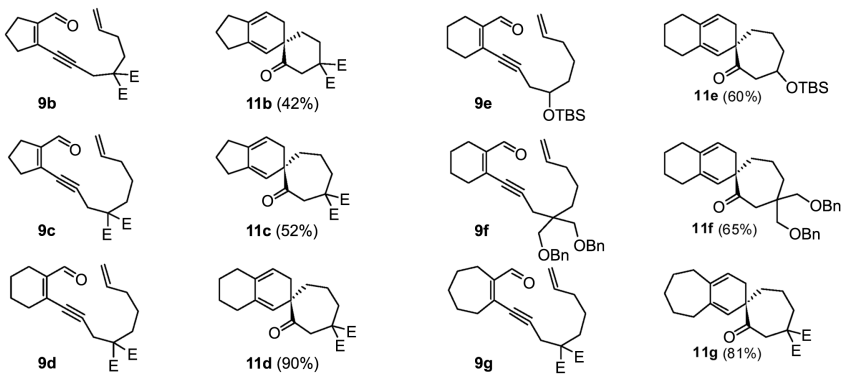

Figure 1. Products 11 of Platinum-catalyzed cyclization, and the corresponding substrates $9 . \mathrm{E}=\mathrm{COOEt}, \mathrm{TBS}=$ tert-butyldimethylsilyl, $\mathrm{Bn}=$ benzyl

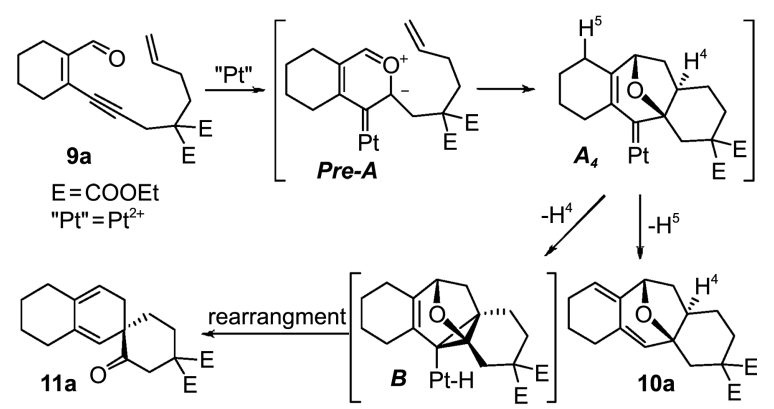

Scheme 2. A possible mechanism for the formation of 10a and 11a

Mechanistically, intermediates pre-A would undergo [3+2] cycloaddition with a pendent double bond to form the Pt-carbene complex $\boldsymbol{A}_{4}$ (Scheme 2). While any basic species, even solvent molecules, could abstract $\mathrm{H}^{5}$ from $\boldsymbol{A}_{4}$ followed by deprotonation to give the product 10a, Pt-carbene complex $\boldsymbol{A}_{4}$, increasing the number of carbon tethers, would be more facile to insert into the tertiary $\mathrm{C}-\mathrm{H}^{4}$ bond to form the fused cyclopropane intermediates $\boldsymbol{B}$ followed by tandem rearrangement to result in spirane 11a.

\section{Conclusion}

Enynals 9 bearing an olefinic pendant were successfully cyclized via Huisgen-type [3+2] cycloaddition to the tetracyclic Pt-carbene complex $\boldsymbol{A}$ which would undergo insertion into a $\mathrm{C}-\mathrm{H}$ bond of the $\beta$-position to afford the fused cyclopropane intermediates and their tandem rearrangement afforded diverse types of spiranes $\mathbf{1 1}$ in good to excellent yields. This convenient methodology can be an useful route to organic chemists for easy access to spirocycle unit such as an effective building block in complex organic synthesis.

\section{Experimental}

General Procedures. In a $5 \mathrm{~mL}$ new test tube, enynals 9a-g $(0.10 \mathrm{mmol}), \mathrm{PtCl}_{2}(10 \mathrm{~mol} \%)$, and dried solvent $(0.5$ $\mathrm{mL}$ ) were charged at 0 . The reaction mixture was kept under argon atmosphere and stirred for 1 to $24 \mathrm{~h}$ in a preheated oil bath (60-120) by monitoring the reactions by TLC periodically. Upon completion, the solvent was removed under vacuum and the crude products were subjected for flash column chromatography to afford the pure products 10a and 11a-g.

Spectroscopic Data of Compounds 10a and 11a-g.

10a: IR $\left(\mathrm{NaCl}, \mathrm{cm}^{-1}\right)$ 1739, 1673, 1598; ${ }^{1} \mathrm{H}$ NMR (400 $\left.\mathrm{MHz}, \mathrm{CDCl}_{3}\right) \delta 5.47(\mathrm{~s}, 1 \mathrm{H}), 5.38(\mathrm{~s} 1 \mathrm{H}), 4.51(\mathrm{t}, J=4.8 \mathrm{~Hz}$, $1 \mathrm{H}), 4.32-4.06(\mathrm{~m}, 4 \mathrm{H}), 2.78(\mathrm{dd}, J=15.0,1.4 \mathrm{~Hz}, 1 \mathrm{H}), 2.31$ $(\mathrm{dd}, J=13.2,2.0 \mathrm{~Hz}, 1 \mathrm{H}), 2.24-2.16(\mathrm{~m}, 3 \mathrm{H}), 2.09-1.99$ (m, $3 \mathrm{H}), 1.79-1.77$ (t, $J=4.4 \mathrm{~Hz}, 2 \mathrm{H}), 1.71-1.54$ (m, 4H), 1.47$1.40(\mathrm{~m}, 1 \mathrm{H}), 1.23(\mathrm{t}, J=7.0 \mathrm{~Hz}, 6 \mathrm{H}) ;{ }^{13} \mathrm{C} \mathrm{NMR}(100 \mathrm{MHz}$, $\left.\mathrm{CDCl}_{3}\right) \delta 172.98,170.94,139.06,131.96,129.11,119.77$, 79.27, 77.45, 61.66, 61.18, 52.01, 43.78, 40.08, 36.09, $30.04,28.98,28.01,25.16,22.79,14.21,14.17$.

11a: IR ( $\left.\mathrm{NaCl}, \mathrm{cm}^{-1}\right) 3470,2935,2864,1730,1447,1367$, 1299, 1251, 1211, 1152; ${ }^{1} \mathrm{H}$ NMR (400 MHz, $\left.\mathrm{CDCl}_{3}\right) \delta 5.43$ 
(s, 1H), $5.40(\mathrm{~s}, 1 \mathrm{H}), 4.23-4.15(\mathrm{~m}, 4 \mathrm{H}), 2.95(\mathrm{~d}, J=14.8$ $\mathrm{Hz}, 1 \mathrm{H}), 2.76(\mathrm{~d}, J=14.4 \mathrm{~Hz}, 1 \mathrm{H}), 2.31-2.20(\mathrm{~m}, 6 \mathrm{H}), 2.02$ $(\mathrm{dd}, J=16.8,4.8 \mathrm{~Hz}, 1 \mathrm{H}), 1.83-1.77(\mathrm{~m}, 1 \mathrm{H}), 1.73-1.66(\mathrm{~m}$, 1H), 1.61-1.46 (m, 4H), 1.27-1.22 (m, 6H); ${ }^{13} \mathrm{C} \mathrm{NMR} \mathrm{(100}$ $\left.\mathrm{MHz}, \mathrm{CDCl}_{3}\right) \delta 208.63,170.84,170.70,136.98,134.02$, $121.94,118.81,62.20,62.13,57.99,48.81,42.42,32.04$, $31.60,31.38,30.96,26.65,24.86,24.77,14.35,14.33$.

11b: IR ( NaCl, cm $\left.{ }^{-1}\right) 3470,2979,1730,1446,1367,1299$, 1249, 1209, 1094; ${ }^{1} \mathrm{H}$ NMR (400 MHz, $\left.\mathrm{CDCl}_{3}\right) \delta 5.49(\mathrm{~s}$, $1 \mathrm{H}), 5.43(\mathrm{~s}, 1 \mathrm{H}), 4.23-4.16(\mathrm{~m}, 4 \mathrm{H}), 2.94(\mathrm{t}, J=14.4 \mathrm{~Hz}$, $1 \mathrm{H}), 2.78-2.69(\mathrm{~m}, 2 \mathrm{H}), 2.78-2.69(\mathrm{~m}, 2 \mathrm{H}), 2.34-2.23(\mathrm{~m}$, $6 \mathrm{H}), 2.11$ (td, $J=17.6,2.4 \mathrm{~Hz}, 1 \mathrm{H}), 1.84-1.64(\mathrm{~m}, 5 \mathrm{H}), 1.27-$ $1.22(\mathrm{~m}, 6 \mathrm{H}) ;{ }^{13} \mathrm{C} \mathrm{NMR}\left(100 \mathrm{MHz}, \mathrm{CDCl}_{3}\right) \delta 208.68$, $170.81,170.71,142.58,138.69,116.99114 .70,62.18$, $62.13,57.92,49.78,42.40,33.17,32.51,31.66,31.02$, 26.28, 25.24, 14.34 .

11c: $\mathrm{IR}\left(\mathrm{NaCl}, \mathrm{cm}^{-1}\right)$ 3514, 2938, 2870, 1731, 1446, 1367 , 1314, 1248, 1201, 1177; ${ }^{1} \mathrm{H}$ NMR (400 MHz, $\left.\mathrm{CDCl}_{3}\right) \delta 5.45$ $(\mathrm{s}, 1 \mathrm{H}), 5.34(\mathrm{~s}, 1 \mathrm{H}), 4.26-4.13(\mathrm{~m}, 4 \mathrm{H}), 3.32(\mathrm{~d}, J=11.6 \mathrm{~Hz}$, $1 \mathrm{H}), 2.98(\mathrm{~d}, J=11.6 \mathrm{~Hz}, 1 \mathrm{H}), 2.85-2.79(\mathrm{~m}, 1 \mathrm{H}), 2.34-2.20$ $(\mathrm{m}, 5 \mathrm{H}), 2.03(\mathrm{td}, J=17.6,2.0 \mathrm{~Hz}, 1 \mathrm{H}), 1.87-1.60(\mathrm{~m}, 7 \mathrm{H})$, $1.26(\mathrm{td}, J=7.2,5.8 \mathrm{~Hz}, 6 \mathrm{H}) ;{ }^{13} \mathrm{C} \mathrm{NMR}\left(100 \mathrm{MHz}, \mathrm{CDCl}_{3}\right)$ $\delta 209.77,171.56,170.90,142.74,138.71,117.50,115.52$, $62.05,61.89,55.38,53.57,42.51,37.17,36.06,32.47$, $31.65,31.00,25.27,20.87,14.33$.

11d: $\mathrm{IR}\left(\mathrm{NaCl}, \mathrm{cm}^{-1}\right) 3470,2981,2935,2862,1732,1707$, 1449, 1367, 1249, 1201; ${ }^{1} \mathrm{H}$ NMR (400 MHz, $\left.\mathrm{CDCl}_{3}\right) \delta 5.41$ $(\mathrm{s}, 1 \mathrm{H}), 5.31(\mathrm{~s}, 1 \mathrm{H}), 4.25-4.15(\mathrm{~m}, 4 \mathrm{H}), 3.31(\mathrm{~d}, J=12 \mathrm{~Hz}$, $1 \mathrm{H}), 2.96(\mathrm{~d}, J=12 \mathrm{~Hz}, 1 \mathrm{H}), 2.68-2.62(\mathrm{~m}, 1 \mathrm{H}), 2.26-2.15$ (m, 5H), 1.98-1.86 (m, 2H), 1.72-1.71 (m, 3H), 1.58-1.50 $(\mathrm{m}, 5 \mathrm{H}), 1.28-1.23(\mathrm{~m}, 6 \mathrm{H}) ;{ }^{13} \mathrm{C} \mathrm{NMR}\left(100 \mathrm{MHz}, \mathrm{CDCl}_{3}\right) \delta$ 209.70, 171.55, 170.94, 137.11, 133.98, 122.51, 119.76, $62.03,61.89,55.19,52.56,42.39,36.36,35.87,31.56$, $31.53,30.93,24.86,24.78,20.83,14.32$.

11e: IR $\left(\mathrm{NaCl}, \mathrm{cm}^{-1}\right)$ 2929, 2856, 1709, 1471, 1461, 1434, 1376, 1360, 1252, 1078; ${ }^{1} \mathrm{H}$ NMR (400 MHz, $\left.\mathrm{CDCl}_{3}\right) \delta 5.41$ $(\mathrm{s}, 1 \mathrm{H}), 5.28(\mathrm{~s}, 1 \mathrm{H}), 3.88-3.81(\mathrm{~m}, 1 \mathrm{H}), 3.02(\mathrm{t}, J=10.2 \mathrm{~Hz}$, $1 \mathrm{H}), 2.75$ (d, $J=16.4 \mathrm{~Hz}, 1 \mathrm{H}), 2.55(\mathrm{dd}, J=12,2.8 \mathrm{~Hz}, 1 \mathrm{H})$, $2.23(\mathrm{~s}, 4 \mathrm{H}), 1.89-1.50(\mathrm{~m}, 10 \mathrm{H}), 1.17-1.11(\mathrm{~m}, 1 \mathrm{H}), 0.88(\mathrm{~s}$, $9 \mathrm{H}), 0.07(\mathrm{~d}, J=6.4 \mathrm{~Hz}, 6 \mathrm{H}) ;{ }^{13} \mathrm{C} \mathrm{NMR}\left(100 \mathrm{MHz} \mathrm{CDCl}_{3}\right) \delta$ 211.34, 136.19, 133.58, 122.94, 119.80, 70.83, 52.22, 49.75, $40.23,35.38,31.50,31.33,30.91,26.15,24.87,24.80$, $19.35,18.45,-4.53$.

11f: IR $\left(\mathrm{NaCl}, \mathrm{cm}^{-1}\right) 3062,3029,2928,2857,1687,1495$, 1452, 1363, 1099, 1027; ${ }^{1} \mathrm{H}$ NMR (400 MHz, $\mathrm{CDCl}_{3}$ ) $\delta 7.32-$ $7.27(\mathrm{~m}, 10 \mathrm{H}), 5.38(\mathrm{~s}, 1 \mathrm{H}), 5.32(\mathrm{~s}, 1 \mathrm{H}), 4.54-4.44(\mathrm{~m}, 4 \mathrm{H})$, 3.37-3.29 (m, 4H), $2.74(\mathrm{~d}, J=11.6 \mathrm{~Hz}, 1 \mathrm{H}), 2.67-2.62(\mathrm{~m}$, $1 \mathrm{H}), 2.45(\mathrm{~d}, J=11.6 \mathrm{~Hz}, 1 \mathrm{H}), 2.24-2.19(\mathrm{~m}, 6 \mathrm{H}), 1.95-1.91$ $(\mathrm{m}, 2 \mathrm{H}), 1.75-1.34(\mathrm{~m}, 7 \mathrm{H}) ;{ }^{13} \mathrm{C} \mathrm{NMR}\left(100 \mathrm{MHz}, \mathrm{CDCl}_{3}\right) \delta$ $213.10,139.15,139.08,136.06,134.05,128.61,128.56$, $127.78,127.71,127.65,123.35,119.62,74.46,73.55,73.50$, $73.29,52.39,42.76,41.28,36.76,33.75,31.99,31.56$, $31.00,24.94,24.87,19.63$.

11g: IR ( $\left.\mathrm{NaCl}, \mathrm{cm}^{-1}\right)$ 3471, 2980, 2923, 2851, 1732, 1708, 1451, 1367, 1249, 1200; ${ }^{1} \mathrm{H}$ NMR (400 MHz, $\left.\mathrm{CDCl}_{3}\right) \delta 5.46$ $(\mathrm{t}, J=4.0 \mathrm{~Hz}, 1 \mathrm{H}), 5.36(\mathrm{~s}, 1 \mathrm{H}), 4.24-4.15(\mathrm{~m}, 4 \mathrm{H}), 3.32(\mathrm{~d}$, $J=12.0 \mathrm{~Hz}, 1 \mathrm{H}), 2.96(\mathrm{~d}, J=12.0 \mathrm{~Hz}, 1 \mathrm{H}), 2.70(\mathrm{dd}, J=$ 17.2, 4.0 Hz, 1H), 2.23-2.19 (m, 6H), 1.95 (dd, $J=17.2$, 4.4 $\mathrm{Hz}, 1 \mathrm{H}), 1.89-1.85(\mathrm{~m}, 1 \mathrm{H}), 1.83-1.78(\mathrm{~m}, 1 \mathrm{H}), 1.76-1.52$ $(\mathrm{m}, 8 \mathrm{H}), 1.26(\mathrm{dd}, J=6.4,7.2 \mathrm{~Hz}, 6 \mathrm{H}) ;{ }^{13} \mathrm{C} \mathrm{NMR}(100 \mathrm{MHz}$, $\left.\mathrm{CDCl}_{3}\right) \delta 209.74,171.57,170.91,141.57,138.32,123.24$, $121.01,62.05,61.90,55.27,52.79,42.39,36.53,35.97$, $35.91,35.76,31.74,31.53,30.81,20.74,14.32$.

Acknowledgments. This work was supported by the research fund of Hanyang University (HY-2007-I) (1|2007 00000006291).

\section{References}

1. (a) Pellissier, H. Adv. Synth. Catal. 2011, 353, 189. (b) Gung, B. W.; Bailey, L. N.; Wonser, J. Tetrahedron Lett. 2010, 51, 2251. (c) Nicolaou, K. C.; Skokotas, G.; Skokotas, S.; Skokotas, H.; Suemune, D.; Nicolaou, C. Angew. Chem. Int. Ed. 1990, 102, 1066. (d) Asao, N.; Sato, K.; Menggenbateer; Yamamoto,Y. J. Org. Chem. 2005, 70, 3682. (e) Dyker, G.; Hildebrandt, D. J. Org. Chem. 2005, 70, 6093. (f) Luo, Y.; Herndon, J. W. Organometallics 2005, 24, 3099. (g) Padwa, A.; Krumpe, K. E.; Gareau, Y.; Chiacchio, U. J. Org. Chem. 1991, 56, 2523. (h) Padwa, A.; Krumpe, K. E.; Zhi, L. Tetrahedron Lett. 1989, 30, 2633. (i) Asao, N.; Aikawa, H.; Yamamoto, Y. J. Am. Chem. Soc. 2004, 126, 7458. (j) Hashmi, A. S. K.; Frost, T. M.; Bats, J. W. J. Am. Chem. Soc. 2000, 122, 11553. (k) Hashmi, A. S. K.; Frost, T. M.; Bats, J. W. Catal. Today 2002, 72, 19. (1) Dankwardt, J. W.; Tetrahedron Lett. 2001, 42, 4237. (m) Hildebrandt, D.; Hüggenberg, W.; Kanthak, M.; Plöger, T.; Müller, I. M.; Dyker, G. Chem. Commun. 2006, 2260. (n) Kusama, H.; Iwasawa, N. Chem. Lett. 2006, 1082. (o) Shin, S.; Gupta, A. K.; Rhim, C. Y.; Oh, C. H. Chem. Commun. 2005, 4429.

2. Saito, K.; Sogou, H.; Suga, T.; Kusama, H.; Iwasawa, N. J. Am. Chem. Soc. 2011, 133, 689-691. (b) Oh, C. H.; Yi, H. J.; Lee, J. H.; Lim, D. H. Chem. Commun. 2010, 46, 2337. (c) Oh, C. H.; Lee, S. M.; Hong, C. S. Org. Lett. 2010, 12, 1308. (d) Ishida, K.; Kusama, H.; Iwasawa, N. J. Am. Chem. Soc. 2010, 132, 8842.

3. (a) Kim, N.; Kim, Y.; Park, W.; Sung, D.; Gupta, A. K.; Oh, C. H. Org. Lett. 2005, 7, 5289.

4. For benzannulation of o-alkynylbenzaldehydes, see: (a) Asao, N.; Aikawa, H.; Yamamoto, Y. J. Am. Chem. Soc. 2004, 126, 7458. (b) Asao, N.; Sato, K.; Menggenbateer; Yamamoto, Y. J. Org. Chem. 2005, 70, 3682. (c) Hashmi, A. S. K.; Frost, T. M.; Bats, J. W. J. Am. Chem. Soc. 2000, 122, 11553. (d) Dankwardt, J. W. Tetrahedron Lett. 2001, 42, 5809. For a mechanistic discussion of this, see: (e) Dyker, G.; Hildebrandt, D. J. Org. Chem. 2005, 70, 6093. f) Kusama, H.; Funami, H.; Iwasawa, N. Synthesis 2007, 2014.

5. For Rh-catalyzed annulations, see: Shin, S.; Gupta, A. K.; Rhim, C. Y.; Oh, C. H. Chem. Commun. 2005, 4429.

6. (a) Oh, C. H.; Lee, J. H.; Lee, S. J.; Kim, J. I.; Hong, C. S. Angew. Chem. Int. Ed. 2008, 47, 7505. (b) Kusama, H.; Funami, H.; Iwasawa, N. Synthesis 2007, 2014. (c) Kusama, H.; Funami, H.; Shido, M.; Hara, Y.; Takaya, J.; Iwasawa, N. J. Am. Chem. Soc. 2005, 127, 2709. (d) Iwasawa, N.; Shido, M.; Kusama, H. J. Am. Chem. Soc. 2001, 123, 5814.

7. Hildebrandte, D.; Ggenberg, W. H.; Kanthak, M.; Plcger, T.; Muller, I. M.; Dyker, G. Chem. Commun. 2006, 2260.

8. Oh, C. H.; Lee, J. H.; Lee, S. M.; Yi, H. J.; Hong, C. S. Chem. Eur. J. 2009, 15, 71 .

9. Oh, C. H.; Ryu, J. H.; Lee, H. I. Synlett 2007, 15, 2337. 